\title{
Elementary Approximation of Exponentials of Lie Polynomials
}

\author{
Frédéric JEAn \& Pierre-Vincent KoselefF \\ Équipe Analyse Algébrique, Institut de Mathématiques \\ Université Paris 6, Case 82 \\ 4 place Jussieu, F-75252 Paris Cedex 05 \\ e-mail : [jean,koseleff]@math.jussieu.fr
}

\begin{abstract}
Let $\mathcal{L}=L\left(x_{1}, \ldots, x_{m}\right)$ be a graded Lie algebra generated by $\left\{x_{1}, \ldots, x_{m}\right\}$. In this paper, we show that for any element $P$ in $\mathcal{L}$ and any order $k, \exp (P)$ may be approximated at the order $k$ by a finite product of elementary factors $\exp \left(\lambda_{i} x_{i}\right)$. We give an explicit construction that avoids any calculation in the Lie algebra.
\end{abstract}

\section{Introduction}

In hamiltonian mechanics, the phase space is governed by an Hamiltonian $h$ and the equations $\dot{z}_{i}=\left\{z_{i}, h\right\}$ where $\{$,$\} is the Poisson bracket. The set of smooth$ functions on the phase space is turned into Lie algebra by considering the Poisson bracket. Integrations of hamiltonian flows by numerical schemes make use of the so-called symplectic integrators that preserve some invariants (see $[8,12,13])$. One will try to approximate the flow $\exp (t\{\cdot, A+B\})$ by composition of the hamiltonian flows of $\exp (t\{\cdot, A\})$ of $A$ and $\exp (t\{\cdot, B\})$ of $B$. These methods are used for their stability in very long-time integration problems. Such integrators may be found by considering universal identities in free Lie algebras (see $[4,5$, $13,14])$.

In control theory, for a control system $\dot{x}=\sum_{i=1}^{m} u_{i}(t) X_{i}(x)$, the classical problem of motion planning is the following (see $[3,6,7]$ ): given two states $p$ and $q$, find $a$ feasible trajectory (i.e. the controls $\left.u_{1}(t), \ldots, u_{m}(t)\right)$ that steers the system from $p$ to a point arbitrarily close to $q$.

Let us assume that $q$ is given as $\exp (X) p$, where $X$ belongs to the Lie algebra generated by the vector fields $X_{i}$. We are interested in the simplest trajectories, those obtained as composition of the flows of the $X_{i}$ 's. The end point of such a trajectory is written $\exp \left(\lambda_{1} X_{i_{1}}\right) \cdots \exp \left(\lambda_{s} X_{i_{s}}\right) p$, that is a product of elementary factors applied to the state $p$. A solution of our problem can then be obtained by approximating the exponential $\exp (X)$ by a product of elementary factors $\exp \left(\lambda X_{i}\right)$.

In this paper we will see such approximations as universal approximations in graded Lie algebra. That is why we will work in free Lie algebras and look for universal identities in free Lie algebras. In Section 1, we will first introduce 
some notations and set our result in Part 1.3. In Section $\mathbf{2}$ we will describe several independent steps of the algorithm and finally we will discuss in Section $\mathbf{3}$ the accuracy of our algorithm by considering some examples and giving some bounds for the complexity. We will finally study the application of our result to the motion planning problem.

\section{Notations and Definitions}

In this section we recall some basic results about Lie algebra. To keep the paper easy to read, we will not go too deeply into the theory and will not present the results in a classical way. For instance we present Lemma 3 as a consequence of the Campbell-Hausdorff Theorem and we will not tell about central integer filtrations (although our result is based only on the properties of these filtrations). For a more classical presentation of this theory we refer to [1, Ch. II] and [10].

\subsection{Notations}

In this paper $A$ will denote an ordered alphabet (possibly endless).

$A^{*}$ is the free monoid generated by $A$ (the set of words). $A^{*}$ is totally ordered with the lexicographic order.

$M(A)$ is the free magma generated by $A$ (the set of parenthesed words). Having defined $M_{1}(A)$ as $A$, we define $M_{n}(A)$ by induction on $n$ :

$$
M_{n}(A)=\bigcup_{p+q=n} M_{p}(A) \times M_{q}(A) \quad \text { and } \quad M(A)=\bigcup_{n \geq 1} M_{n}(A) .
$$

$\mathcal{A}(A)$ is the associative algebra, that is to say the $\mathbb{R}$-algebra of $A^{*}$.

$L(A)$ is the free Lie algebra on $A$. It is defined as the quotient of the $\mathbb{R}$-algebra of $M(A)$ by the ideal generated by the elements $(u, u)$ and $(u,(v, w))+(v,(w, u))+$ $(w,(u, v))$. Its multiplication law [,] is bilinear, alternate and satisfies the Jacobi identity

$$
[x,[y, z]]+[y,[z, x]]+[z,[x, y]]=0 .
$$

An element of $M(A)$ considered as element of $L(A)$ will be called a Lie monomial. Elements of $L(A)$ will be called Lie polynomials.

By setting $[x, y]=x y-y x$ for $x, y \in A$, we have $L(A) \subset \mathcal{A}(A)$. We will also denote by ad $x$, the map $y \mapsto[x, y]$.

$\mathcal{A}(A)$, and hence $L(A)$, are graded by the length (the unique morphism that extends the function $x \mapsto 1$ on $A$ ). For $x \in A^{*}$ (resp. $\left.M(A)\right)|x|$ denotes the length. $L_{n}(A)$ (resp. $\left.\mathcal{A}_{n}(A)\right)$ is the submodule generated by monomials of length $n$. 
We define $\hat{L}(A)$ and $\hat{\mathcal{A}}(A)$ as

$$
\hat{L}(A)=\prod_{n \geq 1} L_{n}(A) \quad \text { and } \quad \hat{\mathcal{A}}(A)=\prod_{n \geq 1} \mathcal{A}_{n}(A) .
$$

We will write $x \in \hat{L}(A)$ as a series $\sum_{n \geq 0} x_{n} . \hat{L}(A)$ is a complete Lie algebra with the Lie bracket

$$
([x, y])_{n}=\sum_{p+q=n}\left[x_{p}, y_{q}\right]
$$

We will also use

$$
\hat{L}_{\geq p}(A)=\prod_{n \geq p} L_{n}(A) \quad \text { and } \quad \hat{\mathcal{A}}_{\geq p}(A)=\prod_{n \geq p} \mathcal{A}_{n}(A) .
$$

\subsection{Exponentials}

One defines the exponential and the logarithm as

$$
\begin{aligned}
\exp : \hat{\mathcal{A}}(A) & \rightarrow 1+\hat{\mathcal{A}}(A) & \log : 1+\hat{\mathcal{A}}(A) & \rightarrow \hat{\mathcal{A}}(A) \\
x & \mapsto \sum_{n \geq 0} \frac{x^{n}}{n !}, & x & \mapsto-\sum_{n \geq 1} \frac{(1-x)^{n}}{n} .
\end{aligned}
$$

They are mutually reciprocal functions and we have (see [1, Ch. II, $\S 5]$ ):

Theorem 1 (Campbell-Hausdorff). For $a, b \in A$, let $H(a, b)$ such that:

$$
\exp (a) \exp (b)=\exp (H(a, b))
$$

Then $H(a, b) \in \hat{L}_{\geq 1}(A)$ and $H_{1}(a, b)=a+b, H_{2}(a, b)=\frac{1}{2}[a, b]$.

Therefore, for $x, y \in \hat{L}$, we have

$$
\exp (H(x, y))=\exp (x) \exp (y) .
$$

and $(H(x, y))_{k}-\left(x_{k}+y_{k}\right) \in L\left(x_{1}, \ldots, x_{k-1}, y_{1}, \ldots, y_{k-1}\right)$, (the free subalgebra of $\hat{L}(A)$ generated by $\left.\left\{x_{1}, \ldots, x_{k-1}, y_{1}, \ldots, y_{k-1}\right\}\right)$

Remark. - $H(x, y)$ is the series $H(a, b)$ evaluated at $x=a$ and $y=b$. The Hausdorff series $H(x, y)$ may be explicitly computed (see [4]). 


\subsection{Setting of the Main Result}

Let us set $\hat{F}=\exp (\hat{L})$ and $\hat{F}_{\geq p}(A)=\exp \left(\hat{L}_{\geq p}(A)\right)$. As exp is bijective, we deduce from the Campbell-Hausdorff Theorem that the sets $\hat{F}$ and $\hat{F}_{\geq p}(A)$ are some groups.

As

$$
\left[\exp \left(\lambda_{1} a_{1}\right) \cdots \exp \left(\lambda_{n} a_{n}\right)\right]^{-1}=\exp \left(-\lambda_{n} a_{n}\right) \cdots \exp \left(-\lambda_{1} a_{1}\right),
$$

the set $G$ generated by the family $\{\exp (\lambda a), \lambda \in \mathbb{R}, a \in A\}$ is a subgroup of $\hat{F}$.

We will show that the elements of $\exp (L(A))$ can be approximated by elements of $G$. Let us first clarify the meaning of approximation. If $P \in L(A)$ is a Lie polynomial, we say that $\alpha=\varphi_{k}(P)$ is a $k$ th-order approximation of $\exp (P)$ if:

$$
\begin{array}{r}
\alpha=\exp \left(\lambda_{1} a_{1}\right) \cdots \exp \left(\lambda_{m} a_{m}\right) \in G, \\
\exp (-P) \alpha \in \hat{F}_{\geq k+1}(A) .
\end{array}
$$

Using Theorem 1, we thus have

$$
\varphi_{k}(P)=\exp (P) \exp \left(R_{\geq k+1}\right)=\exp \left(P+R_{\geq k+1}^{\prime}\right)
$$

where $R_{\geq k+1}$ and $R_{\geq k+1}^{\prime} \in \hat{L}_{\geq k+1}(A)$. Both characterizations will be used in the sequel.

The aim of this paper is to prove the following theorem (Theorem 8):

Theorem. Let $P$ be a Lie polynomial of degree $n$. Then there exists an approximation of $\exp (P)$ at any order $k \geq n$.

Furthermore we will give here an explicit construction of an approximation $\varphi_{k}(P)$. We will use the following steps

1. Define an approximation $\varphi_{n}(\lambda m)$ for any $\lambda \in \mathbb{R}$ and any Lie monomial $m \in M_{n}(A)$. This approximation will have the order $n=|m|$.

2. For any homogeneous Lie polynomial $P$ of degree $n$, define an approximation $\varphi_{n}(P)$ of order $n$.

3. For any homogeneous Lie polynomial, show how to build a $k$ th-order approximation $\varphi_{k}(P)$ from the approximation $\varphi_{n}(P)$. This process will depend only on $n$ and $k$.

4. For any polynomial $P$, show how to get a $k$ th-order approximation. This makes use of the factored product expansion

$$
\exp (P)=\exp \left(P_{1}\right) \cdots \exp \left(P_{k}\right) \exp \left(R_{\geq k+1}\right)
$$

where $P_{i} \in L_{i}(A)$ and $R_{k+1} \in \hat{L}_{\geq k+1}(A)$. This factorization is not given explicitly but may be computed using the Hausdorff series that may also be computed. 


\section{Approximations of Exponential of Lie Polynomials}

Before going more deeply into the method of approximation, we need some results on the groups $\hat{F}$ and $G$.

\subsection{The Groups $\hat{\boldsymbol{F}}$ and $G$}

Let $\alpha \in G$. As element of $\hat{F}_{\geq 1}(A), \alpha$ is written as $\exp \left(\sum_{n \geq 1} x_{n}\right)$, with $x_{n} \in$ $L_{n}(A)$, and the inverse of $\alpha$ is:

$$
\alpha^{-1}=\exp \left(-\sum_{n \geq 1} x_{n}\right)
$$

Given $x \in \hat{L}$, we consider $\exp (\operatorname{ad} x)$ defined as

$$
\exp (\operatorname{ad} x) y=\sum_{i \geq 0} \frac{(\operatorname{ad} x)^{i}}{i !} y
$$

Lemma 2. For $x \in \hat{L}$, we have (see [1])

$$
\exp (x) \exp (y) \exp (-x)=\exp (\exp (\operatorname{ad} x) y) \text {. }
$$

We thus deduce the well-known lemma

Lemma 3. Given $\alpha=\exp (x) \in \hat{F}_{\geq p}(A), \beta=\exp (y) \in \hat{F}_{\geq q}(A)$, we have

$$
\alpha \beta \alpha^{-1} \beta^{-1}=\exp (z) \in \hat{F}_{\geq p+q}(A) \quad \text { and } \quad z_{p+q}=\left[x_{p}, y_{q}\right] .
$$

Proof. - Let $\alpha=\exp \left(\sum_{k \geq p} x_{k}\right), \beta=\exp \left(\sum_{k \geq q} y_{k}\right)$. We have

$$
\begin{aligned}
\alpha \beta \alpha^{-1} \beta^{-1} & =\exp (x) \exp (y) \exp (-x) \exp (-y) \\
& =\exp (\exp (\operatorname{ad} x) y) \exp (-y)=\exp (H(\exp (\operatorname{ad} x) y,-y)) .
\end{aligned}
$$

But

$$
\begin{aligned}
H(\exp (\operatorname{ad} x) y,-y) & \left.=\exp (\operatorname{ad} x) y-y+\sum_{k \geq 2} H_{k}(\exp (\operatorname{ad} x) y,-y)\right) \\
& \left.=[x, y]+\sum_{n \geq 2} \frac{(\operatorname{ad} x)^{n}}{n !} y+\sum_{k \geq 2} H_{k}(\exp (\operatorname{ad} x) y,-y)\right) .
\end{aligned}
$$

For each $n \geq 2,(\operatorname{ad} x)^{n} y \in \hat{L}_{\geq n p+q}(A) \subset \hat{L}_{\geq 2 p+q}(A)$.

For each $k \geq 2, H_{k}(\exp (\operatorname{ad} x) y,-y)=H_{k}(\exp (\operatorname{ad} x) y-y,-y)$. But $\exp (\operatorname{ad} x) y-$ $y \in \hat{L}_{\geq p+q}(A)$, so $H_{k}(\exp (\operatorname{ad} x) y-y,-y) \in \hat{L}_{\geq p+2 q}(A)$. 
For $t \in \mathbb{R}$, we define the morphism of algebra

$$
\begin{aligned}
\phi_{t}: \hat{\mathcal{A}}(A) & \rightarrow \hat{\mathcal{A}}(A) \\
\sum_{n \geq 1} x_{n} & \mapsto \sum_{n \geq 1} t^{n} x_{n} .
\end{aligned}
$$

If $\alpha=\exp \left(\lambda_{1} a_{1}\right) \cdots \exp \left(\lambda_{n} a_{n}\right) \in G \subset 1+\hat{\mathcal{A}}(A)$, then we denote by $\alpha[t]$

$$
\alpha[t]=\phi_{t}(\alpha)=\exp \left(t \lambda_{1} a_{1}\right) \cdots \exp \left(t \lambda_{n} a_{n}\right) \in G .
$$

\subsection{Approximation at the Order $n$}

Lemma 4. Let $x$ be a Lie monomial of length $n$. Then for each $\lambda$ in $\mathbb{R}$ there exists an approximation of $\exp (\lambda x)$ at order $n$.

Proof. - The proof and also the construction of the approximation is given by induction on $|x|$.

If $x \in A, \exp (\lambda x)$ is obviously an approximation of itself at any order. Let us assume that the result is true for the Lie monomials of length $\leq n$ and let $x$ be a monomial of length $n+1$. In a canonical way we can write $x$ as $[a, b]$ with $a$ and $b$ monomials of length $p$ and $q \leq n$ such that $p+q=n+1$. We have also $\lambda x=[\lambda a, b]$ for any $\lambda \in \mathbb{R}$.

By induction hypothesis there exist $\alpha, \beta \in G$ such that:

$$
\begin{aligned}
& \alpha=\exp \left(\lambda a+R_{\geq p+1}\right), R_{\geq p+1} \in \hat{L}_{\geq p+1}(A), \\
& \beta=\exp \left(b+R_{\geq q+1}\right), R_{\geq q+1} \in \hat{L}_{\geq q+1}(A) .
\end{aligned}
$$

We deduce from Lemma 3 that

$$
\gamma=\alpha \beta \alpha^{-1} \beta^{-1}=\exp (\lambda[a, b]+R), R \in \hat{L}_{\geq p+q+1}(A)=\hat{L}_{\geq n+2}(A) .
$$

Thus $\gamma$ is an approximation of $\exp (\lambda x)$ at order $n+1$ and the induction is done.

Lemma 5. Let $P$ and $Q$ be homogeneous Lie polynomials of degree $n \geq 1$ and assume that $\varphi_{n}(P)$ and $\varphi_{n}(Q)$ are approximations of $\exp (P)$ and $\exp (Q)$ at order $n$. Then

$$
\varphi_{n}(P+Q)=\varphi_{n}(P) \varphi_{n}(Q)
$$

is an approximation of $\exp (P+Q)$ at order $n$. 
Proof. - The approximations $\varphi_{n}(P)$ and $\varphi_{n}(Q)$ can be written as

$$
\varphi_{n}(P)=\exp (P+R), \varphi_{n}(Q)=\exp \left(Q+R^{\prime}\right), R, R^{\prime} \in \hat{L}_{\geq n+1}(A) .
$$

Using the Campbell-Hausdorff Theorem, we get $\varphi_{n}(P) \varphi_{n}(Q)=\exp (H(P+$ $\left.R, Q+R^{\prime}\right)$ ), where

$$
H\left(P+R, Q+R^{\prime}\right)=P+Q+\left(R+R^{\prime}\right)+\sum_{k \geq 2} H_{k}\left(P+R, Q+R^{\prime}\right) .
$$

But $R+R^{\prime} \in \hat{L}_{\geq n+1}(A)$ and $H_{k}\left(P+R, Q+R^{\prime}\right) \in \hat{L}_{\geq k n}(A) \subset \hat{L}_{\geq n+1}(A)$ when $k \geq 2$ so there is $R^{\prime \prime} \in \hat{L}_{\geq n+1}(A)$ such that

$$
\varphi_{n}(P) \varphi_{n}(Q)=\exp \left(P+Q+R^{\prime \prime}\right)
$$

is a $n$ th-order approximation of $\exp (P+Q)$.

Corollary 6. If $P$ is an homogeneous Lie polynomial of degree $n \geq 1$, then there exists an approximation of $\exp (P)$ at order $n$.

Proof. - Let $P \in L_{n}(A)$. One can write $P=\sum_{i=1}^{d} \lambda_{i} m_{i}$, where the $m_{i}$ 's are Lie monomials and by induction we get, using Lemma 5

$$
\varphi_{n}(P)=\varphi_{n}\left(\lambda_{1} m_{1}\right) \cdots \varphi_{n}\left(\lambda_{d} m_{d}\right) .
$$

Note that the $m_{i}$ 's are not unique. That proves only the existence of $n$ th-order approximants.

\subsection{Approximations of Homogeneous Lie Polynomials}

Lemma 7. Let $P$ be an homogeneous Lie polynomial of degree $n$. Then, for any $k \geq n$, there exists a kth-order approximation of $\exp (P)$.

Proof. - For a given polynomial $P$ of degree $n$, we proceed by induction on $k$, following Suzuki's idea ([12]). The case $k=n$ has already been done in Corollary 6. Let us assume that, for some $k \geq n+1$, there exists a $(k-1)$ th-order approximation $\alpha=\varphi_{k-1}(P)$, that is $\alpha=\exp \left(P+\sum_{i \geq k} R_{i}\right)$ with $R_{i} \in L_{i}(A)$.

We have to distinguish two cases.

- If $k$ is odd, we set $\gamma=\alpha[t] \alpha[s] \alpha[t]$ and we will show that for some $t$ and $s$, $\gamma$ is a $k$ th-order approximation of $\exp (P)$. From Formula (5) and applying Theorem 1 twice we get

$$
\gamma=\exp \left(\left(2 t^{n}+s^{n}\right) P+\left(2 t^{k}+s^{k}\right) R_{k}+\sum_{i \geq k+1} R_{i}^{\prime}\right) .
$$

By setting

$$
t=\left(2+(-1)^{n} 2^{n / k}\right)^{-1 / n}, s=-2^{1 / k} t,
$$

we have $2 t^{n}+s^{n}=1$ and $2 t^{k}+s^{k}=0$ (notice that $t$ is defined since $k>n$ ). With these values, $\gamma=\varphi_{k}(P)$ is an approximation of $\exp (P)$ at order $k$. 
- If $k$ is even, we set $\gamma=\alpha[u] \alpha[v]^{-1} \alpha[u]$. In the same way as for the odd case, we have

$$
\gamma=\exp \left(\left(2 u^{n}-v^{n}\right) P+\left(2 u^{k}-v^{k}\right) R_{k}+\sum_{i \geq k+1} R_{i}^{\prime \prime}\right),
$$

and we obtain $2 u^{n}-v^{n}=1$ and $2 u^{k}-v^{k}=0$ by setting

$$
u=\left(2-2^{n / k}\right)^{-1 / n}, v=2^{1 / k} u .
$$

\subsection{Approximation of any Lie Polynomial}

Theorem 8. Let $P$ be a Lie polynomial of degree $n$. Then there exists an approximation of $\exp (P)$ at any order $k \geq n$.

Proof. - This theorem will be proved by using the following result ([11])

Lemma 9. Let $P \in L(A)$. Then for all $k \geq 1$ there exists $P_{1} \in L_{1}(A), \ldots, P_{k} \in$ $L_{k}(A)$ and a remainder $R_{\geq k+1} \in \hat{L}_{\geq k+1}(A)$ such that

$$
\exp (P)=\exp \left(P_{1}\right) \cdots \exp \left(P_{k}\right) \exp \left(R_{\geq k+1}\right) .
$$

Remark. - This result is also a variant of the Zassenhaus formula (see [9]).

The factorization (6) is constructed by induction on $k$. If $R_{\geq k+1}=R_{k+1}+R_{k+2}+$ $\cdots$ is the $(k+1)$ th-order remainder, the $(k+2)$ th-order remainder $R_{\geq k+2}$ is given by

$$
\exp \left(R_{\geq k+2}\right)=\exp \left(-R_{k+1}\right) \exp \left(R_{\geq k+1}\right) .
$$

Theorem 1 ensures that $R_{\geq k+2}$ belongs to $\hat{L}_{\geq k+2}(A)$ and, if we set $P_{k+1}=R_{k+1}$, the induction is done.

Let us fix now $k \geq n$ and consider the factorization (6) of $P$. Each term $P_{i}$ is an homogeneous Lie polynomial of degree $i \leq k$. Lemma 7 can then be applied and we set

$$
\begin{aligned}
\alpha & =\varphi_{k}\left(P_{1}\right) \cdots \varphi_{k}\left(P_{k}\right) \\
& =\exp \left(P_{1}\right) \exp \left(R_{\geq k+1}^{1}\right) \cdots \exp \left(P_{k}\right) \exp \left(R_{\geq k+1}^{k}\right) .
\end{aligned}
$$

From Lemma 2, we have, if $R \in \hat{L}_{\geq k+1}(A)$ :

$$
\begin{aligned}
\exp \left(-P_{i}\right) \exp (R) \exp \left(P_{i}\right) & =\exp \left(\exp \left(\operatorname{ad}\left(-P_{i}\right)\right) R\right) \\
& =\exp \left(R^{\prime}\right)
\end{aligned}
$$

where $R^{\prime} \in \hat{L}_{\geq k+1}(A)$ (see Formula (2)). That means that $\exp (R) \exp \left(P_{i}\right)=$ $\exp \left(P_{i}\right) \exp \left(R^{\prime}\right)$ and so, using this identity $k$ times in the expression of $\alpha$, we obtain:

$$
\begin{aligned}
\alpha & =\exp \left(P_{1}\right) \cdots \exp \left(P_{k}\right) \exp \left(R^{\prime}\right) \\
& =\exp (P) \exp \left(-R_{\geq k+1}\right) \exp \left(R^{\prime}\right),
\end{aligned}
$$

where $R^{\prime} \in \hat{L}_{\geq k+1}(A)$. Thus $\alpha$ is an approximation of $\exp (P)$ at order $k$. 


\subsection{Algorithm}

The proof of Theorem 8 is a constructive proof. It allows the construction of an effective approximation of a Lie polynomial. Let us detail the construction of $\varphi_{k}(P), k$ th-order approximation of $\exp (P)$. As an input, we shall give $P_{1}, \ldots, P_{k}$ such that

$$
\exp (P)=\exp \left(P_{1}\right) \cdots \exp \left(P_{k}\right) \exp \left(R_{\geq k+1}\right) .
$$

Each $P_{i}$ is given as an explicit combination of Lie monomials. As output we will have a list $\left\{\left(\lambda_{i}, a_{i}\right)\right\}$ such that

$$
\varphi_{k}(P)=\exp \left(\lambda_{1} a_{1}\right) \cdots \exp \left(\lambda_{m} a_{m}\right) .
$$

1. For $a \in A, \lambda \in \mathbb{R}$ and $k \geq 1$, we set $\varphi_{k}(\lambda a)=\exp (\lambda a)$.

2. For a monomial $x$ of length $n$, we write $x$ as a bracket $[a, b]$ with $|a|=p$ and $|b|=n-p$ (this decomposition need not be unique). We thus define $\varphi_{n}(\lambda x)$ by the induction formula (see Lemma 4):

$$
\varphi_{n}(\lambda x)=\varphi_{p}(\lambda a) \varphi_{n-p}(b) \varphi_{p}(\lambda a)^{-1} \varphi_{n-p}(b)^{-1} .
$$

3. For an homogeneous polynomial $P=\sum_{i=1}^{d} \lambda_{i} x_{i} \in L_{n}(A)$, we set (see Corollary 6):

$$
\varphi_{n}(P)=\varphi_{n}\left(\lambda_{1} x_{1}\right) \cdots \varphi_{n}\left(\lambda_{d} x_{d}\right) .
$$

4. For an homogeneous polynomial $P \in L_{n}(A)$ and $k \geq n+1$, the approximation is defined by the recursion formulae (see Lemma 7):

if $k$ is odd, then $\varphi_{k}(P)=\varphi_{k-1}(P)[t] \varphi_{k-1}(P)\left[-2^{1 / k} t\right] \varphi_{k-1}(P)[t]$,

if $k$ is even, then $\varphi_{k}(P)=\varphi_{k-1}(P)[u] \varphi_{k-1}(P)\left[2^{1 / k} u\right]^{-1} \varphi_{k-1}(P)[t]$

where $t=\left(2+(-1)^{n} 2^{n / k}\right)^{-1 / n}$ and $u=\left(2-2^{n / k}\right)^{-1 / n}$.

5. For a polynomial $P$ given by $P_{1}, \ldots, P_{k}$ such that

$$
\exp (P)=\exp \left(P_{1}\right) \cdots \exp \left(P_{k}\right) \exp \left(R_{\geq k+1}\right),
$$

we set (see Theorem 8):

$$
\varphi_{k}(P)=\varphi_{k}\left(P_{1}\right) \cdots \varphi_{k}\left(P_{k}\right)
$$

\subsection{A Short Example}

Let $P=a+[b, a]$. We have $\exp (P)=\exp (a) \exp ([b, a]) \exp \left(R_{\geq 3}\right)$. Then we find

$$
\varphi_{2}(a)=\exp (a), \varphi_{2}([b, a])=\varphi_{2}(b) \varphi_{2}(a) \varphi_{2}(b)^{-1} \varphi_{2}(a)^{-1}
$$

and thus

$$
\varphi_{2}(P)=\exp (a) \exp (b) \exp (a) \exp (-b) \exp (-a) .
$$

For the same polynomial $P=a-[a, b]$, we found a shorter solution

$$
\varphi_{2}(P)=\exp (b) \exp (a) \exp (-b) .
$$




\section{Estimations and Example}

In this section we will discuss the shape of the output of our algorithm. We will first estimate the number of elementary factors in the approximation (an elementary factor is a term $\exp (\lambda a)$, with $\lambda \in \mathbb{R}, a \in A)$. Then we will show how one can reduce this complexity.

\subsection{Complexity}

Let $P$ be a Lie polynomial of degree $n$ and an integer $k \geq n$. We assume that we know the decomposition (6)

$$
\exp (P)=\exp \left(P_{1}\right) \cdots \exp \left(P_{k}\right) \exp \left(R_{\geq k+1}\right)
$$

where each polynomial $P_{i}=\sum_{j=1}^{d_{i}} \lambda_{j} x_{j}$ in $L_{i}(A)$ is given as linear combination of Lie monomials.

Although $P_{1}, \ldots, P_{k}$ are unique, their decomposition into sum of monomials is not. The reader must notice that our algorithm will start with the given of the $d_{i}$ 's, the $x_{j}$ 's and $\lambda_{j}$ 's as inputs and that it avoids any calculation in the free Lie algebra. Here we are not concerned with the problem of the decomposition of Lie polynomials in some particular basis. For instance there is no a priori bound for the $d_{i}$ 's (an upper bound could have been given by the dimension of $L_{i}(A)$ that satisfies the Witt formula: $\left.\sum_{d \mid n} d \operatorname{dim}\left(L_{d}(A)\right)=|A|^{n}\right)$.

Under these hypothesis, our algorithm gives a $k$ th-order approximation $\varphi_{k}(P)$. Let $l_{k}(P)$ be the number of elementary factors in $\varphi_{k}(P)$. We will give an upper bound of $l_{k}(P)$ with respect to $d_{1}, \ldots, d_{k}$. We give this estimation by following the step numbers of Part 2.5.

- For $a \in A$ and $\lambda \in \mathbb{R}$, we have obviously $l_{k}(\lambda a)=1$ (see step 1 ).

- If $x_{n}$ is a monomial of length $n$, then, with the notations of step 2, we have:

$$
l_{n}\left(\lambda x_{n}\right) \leq 2\left(l_{p}\left(\lambda a_{p}\right)+l_{q}\left(b_{q}\right)\right) .
$$

By induction on $n$ we see that

$$
l_{n}\left(\lambda x_{n}\right) \leq 3 \times 2^{n-1}-2 .
$$

- For an homogeneous Lie polynomial $P_{i}=\sum_{j=1}^{d_{i}} \lambda_{j} x_{j} \in L_{i}(A)$, step 3 implies that

$$
l_{i}\left(P_{i}\right) \leq 3 d_{i} \times 2^{i-1} .
$$

- Therefore, we have, by using step 4:

$$
l_{k}\left(P_{i}\right) \leq 3^{k-i+1} d_{i} 2^{i-1}=3^{k}\left(\frac{2}{3}\right)^{i-1} d_{i}
$$


- Finally, we get

$$
l_{k}(P) \leq 3^{k} \sum_{i=1}^{k}\left(\frac{2}{3}\right)^{i-1} d_{i} \leq 3^{k+1} \max \left\{d_{i}\right\} .
$$

Remark. - If $P=a+b$ it is known (see $[14,4])$ that $l_{2 k}(a+b)=2^{k+1}-1$ for $k \leq 4$, that is an exponential bound.

We know better estimates in some particular cases. If $P=0$, it has been shown ([2]) that $k \leq l_{k}(P) \leq k^{2}$, that is a polynomial bound. It implies that for any $k$, there exists a Lie polynomial $P_{k} \in L_{k}(A)$ such that $\varphi_{k}\left(P_{k}\right)$ is a product of less than $k^{2}$ factors. This is also far less than the sum of dimensions $\sum_{i=1}^{k} \operatorname{dim}\left(L_{i}(A)\right)$.

These two examples and example 2.6 show that the minimal number of factors of $\varphi_{k}(P)$ depends on the polynomial $P$ and on its decomposition into monomials. In this paper we will not seek minimal approximants (this question is considered in $[5,14]$ and in the remark at the end of Part 3.3).

\subsection{Improvements}

Our construction is certainly not optimal. We can then improve it a lot. Let us notice that the steps of the construction are independent of each other. Each step can then be improved separately. We will discuss now about some possible modifications (we refer to the step numbers of Part 2.5).

We present first a simple improvement of step 4 . When $k$ and $n$ have not the same parity, the recursion formula for $\varphi_{k}(P)$, can be replaced by:

if $n$ is even and $k$ odd, then $\varphi_{k}(P)=\varphi_{k-1}(P)\left[2^{-1 / n}\right] \varphi_{k-1}(P)\left[-2^{-1 / n}\right], \quad(9)$

if $n$ is odd and $k$ even, then $\varphi_{k}(P)=\varphi_{k-1}(P)\left[2^{-1 / n}\right] \varphi_{k-1}(P)\left[-2^{-1 / n}\right]^{-1}$.

This formula allows to reduce the theoretical bound (7) (and then the bound (8)) for the size of the approximation, since it has two factors instead of three. When $n$ and $k$ have the same parity, there is no such formula with two factors.

We are now interested in step 2. We have chosen in our algorithm to write $\lambda x$ as $[\lambda a, b]$. The idea was: if $\lambda$ is an integer, then the elementary factors of $\varphi_{n}(\lambda x)$ will use only integer. However we can see on examples that, with this construction, there are no cancellations between the factors of $\varphi_{n}(\lambda x)$ (we call cancellation the occurrence of a product $\exp (y) \exp (-y))$. For example, we get

$$
\varphi_{2}([x, y])=\exp (x) \exp (y) \exp (-x) \exp (-y)
$$

and we will test our algorithm on $\varphi_{4}(\lambda[[x, y],[x, y]])$. For $\lambda=1$, algorithm produces

$$
\varphi_{4}([[x, y],[x, y]])=1
$$

and for $\lambda=2$, it gives 


$$
\begin{gathered}
\varphi_{4}(2[[x, y],[x, y]])=\exp (2 x) \exp (y) \exp (-x) \exp (-y) \exp (-x) \exp (y) \\
\exp (2 x) \exp (-y) \exp (-x) \exp (y) \exp (-x) \exp (-y) .
\end{gathered}
$$

Thus we see that the cancellations are not preserved by the algorithm.

To avoid this kind of problem we can use another method. We first construct $\varphi_{n}(x)$. Then, if $x$ is a Lie monomial of length $n$, we get

$$
\varphi_{n}(|\lambda| x)=\varphi_{n}(x)\left[|\lambda|^{1 / n}\right] .
$$

Finally we have $\varphi_{n}(\lambda x)=\varphi_{n}(|\lambda| x)^{\sigma}$, where $\sigma= \pm 1$ is such that $\lambda=\sigma|\lambda|$. This formula allows to keep cancellations from $\varphi_{n}(x)$ to $\varphi_{n}(\lambda x)$.

This construction does not give theoretical improvement for the size of the approximation: it does not reduce the bounds (7) and (8). But in practice, it gives often an approximation with less factors. On the other hand, it makes use of algebraic numbers even if $\lambda$ is an integer.

\subsection{Example}

Let $P=a+[b, a]+[a,[b, a]]$. Let us compute the approximation $\varphi_{3}(P)$.

We first show how to get the expansion $(6)$ of $\exp (P)$ at the order 3 .

$$
\begin{aligned}
\exp (P) & =\exp (a+[b, a]+[a,[b, a]]) \\
\exp (-a) \exp (P) & =\exp \left([b, a]+[a,[b, a]]-\frac{1}{2}[a,[b, a]]+R_{\geq 4}\right) \\
\exp (-[b, a]) \exp (-a) \exp (P) & =\exp \left(\frac{1}{2}[a,[b, a]]+R_{\geq 4}^{\prime}\right)
\end{aligned}
$$

We thus deduce that $P_{1}=a, P_{2}=[b, a], P_{3}=\frac{1}{2}[a,[b, a]]$.

Algorithm starts now by computing $\varphi_{3}\left(P_{i}\right)$.

- We get from the previous example (Part 2.6)

$$
\varphi_{3}\left(P_{1}\right)=\exp (a), \varphi_{2}\left(P_{2}\right)=\exp (b) \exp (a) \exp (-b) \exp (-a) .
$$

From Formula (9) we obtain $\varphi_{3}\left(P_{2}\right)=\varphi_{2}\left(P_{2}\right)\left[\frac{1}{\sqrt{2}}\right] \varphi_{2}\left(P_{2}\right)\left[\frac{-1}{\sqrt{2}}\right]$ and then:

$$
\begin{gathered}
\varphi_{3}\left(P_{2}\right)=\exp \left(\frac{1}{\sqrt{2}} b\right) \exp \left(\frac{1}{\sqrt{2}} a\right) \exp \left(\frac{-1}{\sqrt{2}} b\right) \exp \left(\frac{-1}{\sqrt{2}} a\right) \exp \left(\frac{-1}{\sqrt{2}} b\right) \exp \left(\frac{-1}{\sqrt{2}} a\right) \\
\exp \left(\frac{1}{\sqrt{2}} b\right) \exp \left(\frac{1}{\sqrt{2}} a\right)
\end{gathered}
$$

- For $P_{3}$, we have $\varphi_{3}\left(P_{3}\right)=\varphi_{1}\left(\frac{1}{2} a\right) \varphi_{2}([b, a]) \varphi_{1}\left(\frac{1}{2} a\right)^{-1} \varphi_{2}([b, a])^{-1}$, that is:

$$
\begin{gathered}
\varphi_{3}\left(P_{3}\right)=\exp \left(\frac{1}{2} a\right) \exp (b) \exp (a) \exp (-b) \exp (-a) \exp \left(-\frac{1}{2} a\right) \exp (a) \exp (b) \\
\exp (-a) \exp (-b) .
\end{gathered}
$$

Noticing that $\exp (-a) \exp \left(-\frac{1}{2} a\right) \exp (a)=\exp \left(-\frac{1}{2} a\right)$ we get:

$$
\varphi_{3}\left(P_{3}\right)=\exp \left(\frac{1}{2} a\right) \exp (b) \exp (a) \exp (-b) \exp \left(-\frac{1}{2} a\right) \exp (b) \exp (-a) \exp (-b) .
$$


The approximation $\varphi_{3}(P)$ is then given by $\varphi_{3}(P)=\varphi_{3}\left(P_{1}\right) \varphi_{3}\left(P_{2}\right) \varphi_{3}\left(P_{3}\right)$. Taking together the end term of $\varphi_{3}\left(P_{2}\right)$ and the first term of $\varphi_{3}\left(P_{3}\right)$, we get:

$$
\begin{gathered}
\varphi_{3}(P)=\exp (a) \exp \left(\frac{1}{\sqrt{2}} b\right) \exp \left(\frac{1}{\sqrt{2}} a\right) \exp \left(\frac{-1}{\sqrt{2}} b\right) \exp \left(\frac{-1}{\sqrt{2}} a\right) \exp \left(\frac{-1}{\sqrt{2}} b\right) \exp \left(\frac{-1}{\sqrt{2}} a\right) \\
\exp \left(\frac{1}{\sqrt{2}} b\right) \exp \left(\frac{1+\sqrt{2}}{\sqrt{2}} a\right) \exp (b) \exp (a) \exp (-b) \exp \left(-\frac{1}{2} a\right) \exp (b) \exp (-a) \exp (-b) .
\end{gathered}
$$

Remark. - As pointed out at the end of Part 3.1, we could find a shorter solution. If we calculate $\exp (A b) \exp (B a) \exp (C b) \exp (D a) \exp (E b)=\exp (P)$, we find up to order 3 :

$$
\begin{aligned}
& P=(D+B) a+(E+C+A) b+ \\
& +\frac{1}{2}((A-C) B-(D+B) E+(A+C) D)[a, b] \\
& +\frac{1}{12}\left((D+B)^{2} E+(A+C) D^{2}+(2 A B-4 B C) D+B^{2} C+A B^{2}\right)[a,[a, b]] \\
& +\frac{1}{12}\left((B+D) E^{2}+(-4(A+C) D+2 B C-4 A B) E+(A+C)^{2} D\right)[[a, b], b]
\end{aligned}
$$

We thus find a five-factors third-order approximant for $P=a+[b, a]+[a,[b, a]]$, by setting

$$
B=\frac{1}{2}(1+\varepsilon \sqrt{65}), D=\frac{1}{2}(1-\varepsilon \sqrt{65}), C=-\frac{1}{8}, E=\frac{B+4}{8}, A=-\frac{B+3}{8} .
$$

This method makes use of calculations in the free algebra and requires to solve polynomial system solving. It could not be so easely generalized to higher orders.

\section{Application to Control Theory}

The use of our approximations in control theory (in particular for the motion planning problem) will be discussed in a next paper. We give here an example of such application.

Let $X_{1}, \ldots, X_{m}$ be vector fields on $\mathbb{R}^{n}$ and $(\Sigma)$ the control system

$$
\dot{x}=\sum_{i=1}^{m} u_{i}(t) X_{i}(x) .
$$

Let $\mathcal{L}\left(X_{1}, \ldots, X_{m}\right)$ be the Lie algebra generated by $X_{1}, \ldots, X_{m}$. For a given $X$ in $\mathcal{L}\left(X_{1}, \ldots, X_{m}\right)$, we consider the problem of "approximating" a point lying on the flow of $X$. More precisely, the problem is to find a trajectory steering the system from a point $p$ to a point $\exp \left(t X+o\left(t^{k}\right)\right) p\left(o\left(t^{k}\right)\right.$ denotes here a vector field with a norm in $\left.o\left(t^{k}\right)\right)$.

Any composition of flows of the $X_{i}$ 's is a trajectory of the system $(\Sigma)$. The end-point of such a trajectory is written $\exp \left(\lambda_{1} X_{i_{1}}\right) \cdots \exp \left(\lambda_{s} X_{i_{s}}\right) p$. Therefore sequences $\lambda_{j}$ and $X_{i_{j}}$ such that

$$
\exp \left(\lambda_{1} X_{i_{1}}\right) \cdots \exp \left(\lambda_{s} X_{i_{s}}\right) p=\exp \left(t X+o\left(t^{k}\right)\right) p
$$


give a solution to our problem.

We first translate the problem in the context of the free Lie algebras. Let $L(A)$ be the free Lie algebra generated by an alphabet $A$ of $m$ elements. We denote by $o\left(t^{k}\right)$ an element $t^{k} R(t)$ in $\hat{L}(A)$, with $R(t) \in \hat{L}(A)$ for each $t$, and $R(0)=0$. Using the universal Lie algebra morphism $\phi: L(A) \rightarrow \mathcal{L}\left(X_{1}, \ldots, X_{m}\right), a_{i} \mapsto X_{i}$, any relation in the free Lie algebra like

$$
\exp \left(\lambda_{1} a_{1}\right) \cdots \exp \left(\lambda_{s} a_{s}\right)=\exp \left(t P+o\left(t^{k}\right)\right)
$$

will give a relation $(11)$ in $\mathcal{L}\left(X_{1}, \ldots, X_{m}\right)$.

That is why we will establish universal approximations $(12)$ in $L(A)$. Next example shows how it can be done.

\subsection{Example}

Let $P=a+[b, a]+[a,[b, a]]$ (the same polynomial as in Part 3.3) and let us try to build an approximation in $o(t)$. We first compute the expansion (6) of $\exp (t P)$ at order 3. Formula (10) gives

$$
\exp (-t[b, a]) \exp (-t a) \exp (t P)=\exp \left(\left(t-\frac{t^{2}}{2}\right)[a,[b, a]]+R_{\geq 4}^{\prime}(t)\right)
$$

where $R_{\geq 4}^{\prime}(t)=t^{2} R^{\prime}(t)$. We thus deduce that

$$
\exp (t P)=\exp (t a) \exp (t[b, a]) \exp (t[a,[b, a]]) \exp (o(t)) .
$$

Thus the expansion (6) at order 3 gives only an approximation "in $o(t)$ ". In order to get an approximation "in $o\left(t^{2}\right)$ ", we should go until sixth-order in decomposition 6 .

We would like to convince the reader that

$$
\alpha=\varphi_{1}(a)[t] \varphi_{2}([b, a])\left[t^{1 / 2}\right] \varphi_{3}([a,[b, a]])\left[t^{1 / 3}\right]
$$

is an approximation of $\exp (t P)$ in the form (12).

This construction could be generalized to any Lie polynomial $P$. We would have to refine Lemma 9 and then use the approximations $\varphi_{k}(P)$ for the homogeneous Lie polynomials. This will be detailed in a next paper.

\section{$5 \quad$ References}

[1] Bourbaki, N., Groupes et algèbres de Lie, Éléments de Mathématiques, Hermann, Paris, 1972

[2] Falbel, E., Koseleff, P.-V., Parallelograms, Submitted to "Formal Power Series and Combinatorics" (1996)

[3] Jacob, G., Motion Planning by piecewise constant or polynomial inputs, Proceedings of the IFAC Nonlinear Control Systems Design Symposium (1992) 
[4] Koseleff, P.-V., Relations among Formal Lie Series and Construction of Symplectic Integrators, AAECC'10 proceedings, Lect. Not. Comp. Sci. 673 (1993)

[5] Koseleff, P.-V., Exhaustive Search of Symplectic Integrators Using Computer Algebra, Fields Institute Communications 10 (1996)

[6] Lafferriere, G., Sussmann H., Motion Planning for controllable systems without drift, Proceedings of the 1991 IEEE International Conference on Robotics and Automation (1991)

[7] Laumond, J.P., Nonholonomic Motion Planning via Optimal Control, Algorithmic Foundations of Robotics (1995)

[8] MacLachlan, R. I., On the numerical integration of ordinary differential equations by symmetric composition methods, SIAM J. Sci. Comp. 16(1) (1995), 151-168

[9] Magnus et al., Combinatorial Group Theory: Presentation of Groups in Terms of Generators and Relations, J. Wiley \& Sons, 1966

[10] Reutenauer, C., Free Lie algebras, Oxford Science Publications, 1993

[11] Steinberg, S., Lie Series, Lie Transformations, and their Applications, in Lie Methods in Optics, Lec. Notes in Physics 250 (1985)

[12] Suzuki, M., General Theory of higher-order decomposition of exponential operators and symplectic integrators, Physics Letters A 165 (1992), 387-395

[13] Suzuki, M., General nonsymetric higher-order decompositions of exponential operators and symplectic integrators, Physic Letters A 165 (1993), 387-395

[14] Yoshida, H., Construction Of Higher Order Symplectic Integrators, Ph. Letters A 150, (1990), 262-268 